\title{
L-LACTATE MONOOXYGENASE, PROPOSED ACTIVE SITE STRUCTURE AND MECHANISM
}

\author{
Sandro Ghisla* and Vincent Massey $\ddagger$ \\ *Faculty of Biology, University of Konstanz, D-7750 Konstanz, FRG \\ $\ddagger$ Department of Biological Chemistry, Univ. of Michigan, Ann Arbor, Mi. 48109 USA,
}

\section{Introduction}

L-Lactate Oxidase (L-lactate-2-monooxygenase, abbreviated as LO) from Mycobacterium smegmatis has been extensively studied, both with respect to its physicochemical characteristics and its reaction mechanism. These studies provided the basis for several widely accepted concepts of flavoprotein catalysis, including the the mechanism of decarboxylation of $\alpha$-ketcacids, the mechanism of activation of substrates via carbanion formation, the transfer of redox equivalents between substrate and flavin via covalent intermediates. Further detailed knowledge of the enzyme reaction mechanism, and the role of specific amino acid residues in catalysis, has been hampered by the lack of the threedimensional structure. However, the primary structure of $L O$ has been solved recently [1] and found to have considerable homology with spinach glycollate oxidase (GO) and with yeast lactate dehydrogenase (flavocytochrome $b_{2}, b_{2}$ ), which also catalyze dehydrogenation of $\alpha$-hydroxyacids and are believed to share mechanistic similarities. The three-dimensional crystal structures of these enzymes are now available [2,3]. Based on the similarity in protein structures around the flavin in these two enzymes, and strictly conserved residues in the primary structure of $\mathrm{LO}$, we have used these data to interpret in molecular terms the reaction mechanism of $\mathrm{LO}$, and interpret to the effect of interaction of specific amino acid residues with the flavin on the physicochemical properties of the flavoenzyme.

\section{Structural Comparisons of Lactate Oxidase with Spinach Glycollate Oxidase and Ycast Flavocytochrome $b_{2}$.}

The subunit sequence of 393 amino acids of LO shows considerable homology with the sequences of $\mathrm{GO}$ and $\mathrm{b}_{2}[1,4]$. In the sequence comparison 69 residues show identity between all three enzymes. Included among these are residues which are involved in binding to the FMN side chain or isoalloxazine ring, or residues implicated in substrate binding and catalysis. Thus, Arg 413 and Arg433 form salt linkages to the phosphate ofFMN, Asp409 is H-bonded with the FMN ribityl-3'- 
$\mathrm{OH}$ and Lys349 is H-bonded with the ribityl-2'-OH in $\mathrm{b}_{2}$. Exactly the same functions in the $\mathrm{GO}$ crystal structure are ascribed to the homologous residues Arg289, Arg309, Asp284 and Lys230 [3]. With LO the existence of the same homologous residues, Arg324, Arg344, Asp320 and Lys266, suggests strongly a very similar binding of FMN. Similar interactions are also seen between the protein and isoalloxazine ring, with $\mathrm{O}(2)$ (hydrogen bond with Thr 155 in GO, Thr280 in b2 and presumably Thr1 78 in LO). Interaction of $\mathrm{O}(4)$ with $\mathrm{Gln} 127$ and Tyr129 in GO is paralleled by the same interaction with Gln 252 and Tyr 254 in $b_{2}$ and presumably by the equivalent Gln 150 and Tyr 152 in LO. Among the amino acid functions, which are assumed to be part of the catalytic machinery the conservation is impressive, as shown in figure 1 .

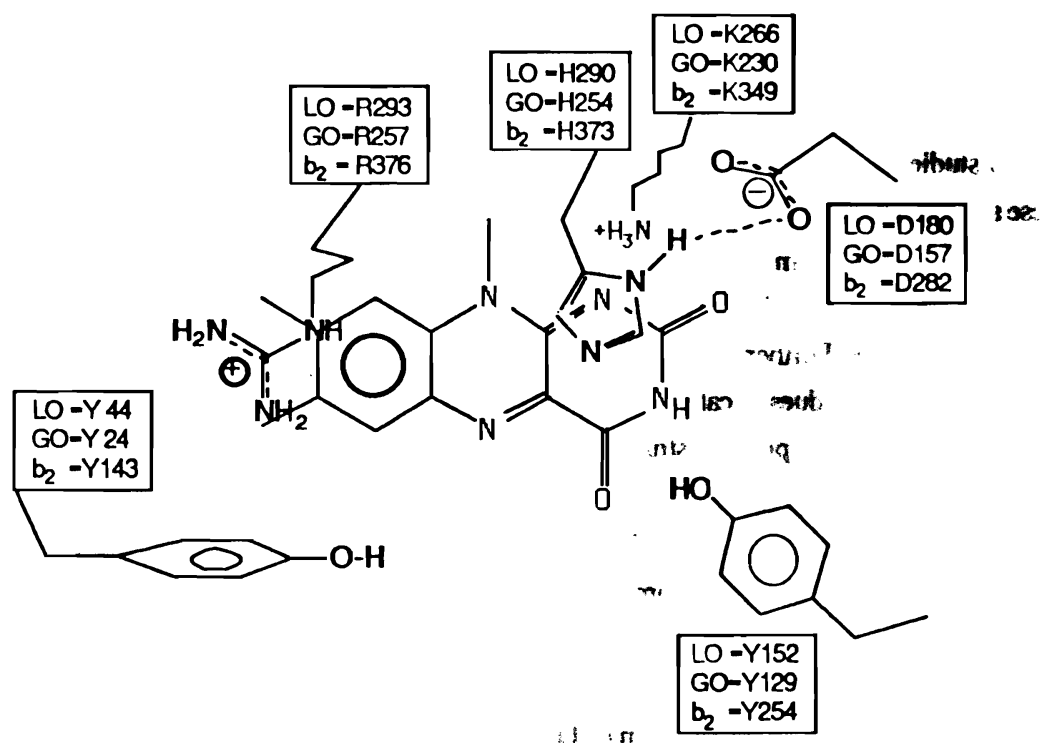

Figure 1: Comparison of active center functional groups for lactate oxidase (LO), glycollate oxidase (GO) and flavocytochrome b2 (b2).

Binding of the substrate in the active site appears to be similar in all three enzymes. From the crystal structures it is known that product or competitive inhibitors are bound on the Si-side of the flavin $[2,3]$; the same side was found with LO in stereospecificity studies employing 8-OH-5-deaza$\mathrm{FMNH}_{2}[5,6]$. With $b_{2}$ the carboxylate of the substrate is held in a salt bridge with Arg 376 and by a hydrogen bond with the hydroxyl of Tyr $143[2,7]$. The same interactions are to be expected in the crystal structure of GO with Arg 257 and Tyr 24 and also presumably in LO where the same functions would be fulfilled by the strictly homologous residues Arg293 and Tyr44. All three proteins contain a strictly conserved histidine residue (His 373 in $b_{2}$, His 254 in GO and presumably 
ITis290 in LO) which is believed to be the active site base responsible for the abstraction of the substrate $\alpha$-hydrogen as a proton to form the carbanion species which then transfers its electrons to the flavin. In both $b_{2}$ and GO there is an aspartate residue (Asp 282 in $b_{2}$ and Asp 157 in GO) with its carboxylate close to $\mathrm{N}(1)$ of this histidine residue, and ascribed a typical charge relay role. An homologous $A$ sp 180 is found in LO. Finally a tyrosine residue located on the substrate binding side of the flavin (Tyr254 in b2, Tyr129 in GO and Tyr 152 in LO) appears to be strictly conserved, and has been thought possibly to play an important role in catalysis, since in both $b_{2}$ and $G O$, the oxygen atom of the tyrosine hydroxyl is in hydrogen bond distance to the substrate $\alpha-\mathrm{OH}$, and thus may serve to facilitate the removal of the proton from the substrate hydroxyl to form the final keto acid product. However, in the Tyr $254 \mathrm{Phe}$ mutant of $b_{2}$ there is $2 \%$ residual catalytic activity [8] and the GO Tyr 129Phe mutant has approximately $10 \%$ the catalytic activity of the wild type enzyme [9].

\section{Catalysis and role of functional groups}

Although a three dimensional structure does not yet exist for $\mathrm{LO}$ the homologies discussed above, and the similarities of the chemical reactions catalzyed (see discussions in $[4,7$ ) allow the deduction that the three enzymes (LO, GO and $b_{2}$ ) work by the same basic chemical mechanism. The mode of substrate binding can be derived from the three dimensional structures of the active center published for $\mathrm{GO}$ and $\mathrm{b}_{2}$. In figure 2 we have simply placed the substrate lactate in the active center cavity such as to yield an optimal interaction. The same procedure was carried through in a three dimensional system using the conventional stick-frame display and space filling models (not shown) from which it can be confirmed that the two dimensional picture of figure 2 is reasonable. In the model of figure 2 catalysis will be initiated by abstraction of the $\alpha$-hydrogen as a proton. The base involved has been proposed to be a histidine [2] (His 290 in LO, see figure 1), which is linked to an aspartic acid (Asp 280 ), in a charge relay system. Tyr 44 interacts with the carbonyl or the carboxylate and this might contribute to the acidification of the $\alpha$-hydrogen. Tyr 152 is shown to interact with the substrate $\alpha$ $\mathrm{OH}$ group and might help in keeping it in the proper orientation. A question which is often brought up in the context of the reaction mechanism, refers to the mode of stabilization of the carbanion formed, i.e. of the transition state. We have proposed oxalate to be a transition state analog $[10,11]$ binding in a bidentate manner to a basic group serving in the fixation of substrate (Arg293 in LO), and to the protonated base, which abstracts the $\alpha$-hydrogen, in this case His290 [4]. Oxalate (dianionic form) can be fitted nicely into the active œnter of models of $\mathrm{LO}$ where it interacts with the two positively charged amino acids Arg293, His290, and the two tyrosines 44 and 152. (Full) delocalisation of the negative charge of the substrate carbanion to the carbonyl of the $C(1)$-carboxylate would form a planar molecule as shown in figure 3, in which the $\mathrm{C} \alpha$ negative charge is stabilized by the interaction with Tyr 44. This could go as far as to reach a complete transfer in which Tyr44 is in its anionic form, as shown. Clearly in a transition state such an extreme (mesomeric) form is not required to exist, and transfer of charge might be only partial. In any case the interaction described appears appropriate for such a transition state stabilization interaction. Important mechanistic 


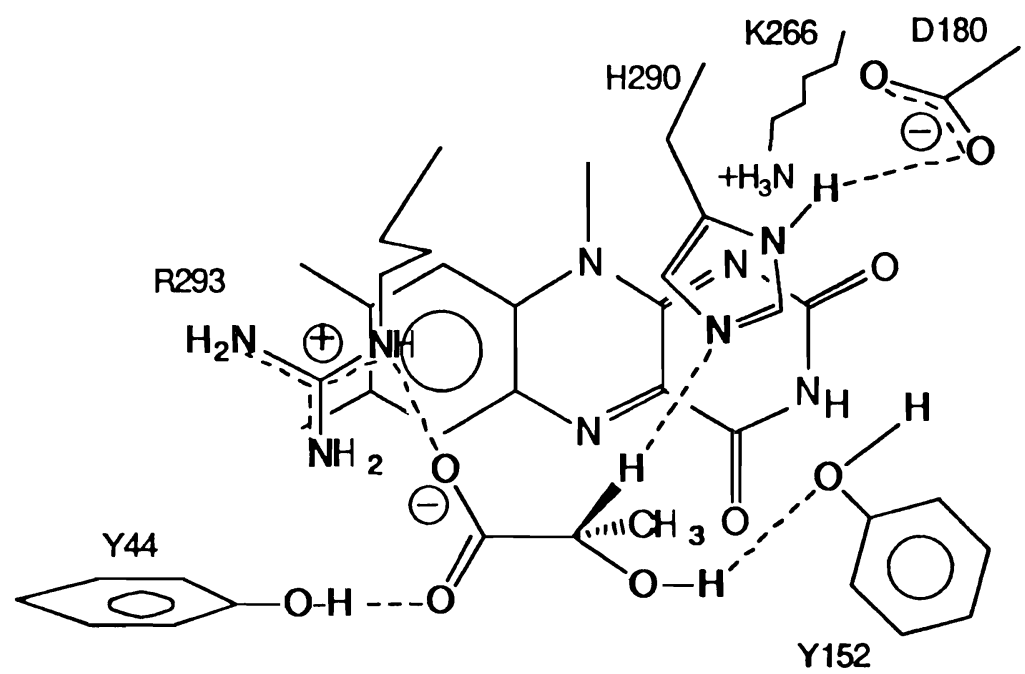

Figure 2: Possible orientation of lactate, isoalloxazine and functional groups involved in binding and catalysis in the Michaelis complex of lactate oxidase.

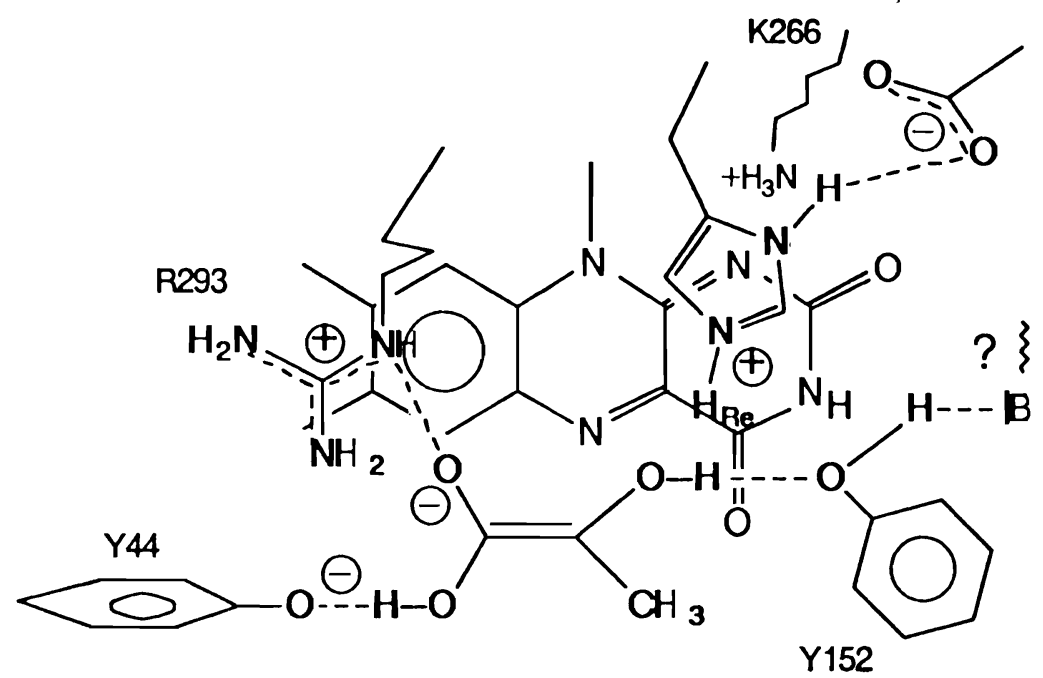

Figure 3 Possible mode of stabilization of (a mesomeric form of) the carbanion generated by abstraction of the substrate $\alpha$-hydrogen as a proton by His 290 . The protonated form of Tyr44 is envisaged to form a hydrogen bond to the carbonyl of substrate

information on lactate oxidase was obtained from the study of its reaction with glycollate [12-14]. With the latter, in contrast to the case of L-lactate, both hydrogens ( $\mathrm{Re}$ and $\mathrm{Si}$ ) were shown to 
undergo abstraction, although at vastly different rates [13]. The salient difference between the reaction with $\mathrm{L}$-lactate and glycollate, however, is the occurrence with the latter of two different intermediates arising from abstraction of the $\alpha$-hydrogen, and before (or concomitant with) formation of the complex of reduced enzyme and glyoxylate. These two intermediates were shown $[13,14]$ to be derived from abstraction, respectively of the $\mathrm{Re}$ - and $\mathrm{Si}$ hydrogen of glycollate, and thus most probably have enantiomeric structures. Most importantly the one derived from rupture of the Sihydrogen bond is relatively stable. Its structure was shown to be that of an N(5) glycollyl flavin adduct as shown in figure 4A. By analogy and by comparison of the corresponding properties the second intermediate was proposed to be that derived from abstraction of the Re-hydrogen (which corresponds stereochemically to the $\alpha-\mathrm{H}$ in L-Lactate) $[13,14]$. The two sets of reactions leading to the two intermediates are shown in figures $4 \mathrm{~A}$ and $4 \mathrm{~B}$. The difference in stability between the two adducts can be nicely rationalized by comparing the three dimensional orientation of the $\alpha$ substituents and their interactions with Tyr 152. In the case of the labile adduct (figure 4A) the inferaction is shown to facilitate the fragmentation of the adduct, while it is absent in the stable adduct (figure 4B). This proposal requires an active role of Tyr152 in catalysis. In fact, in a Tyr254Phe mutant of $b_{2}$ and in a similar one (Tyr139Phe) of GO the activity is reduced to 2 and $10 \%$ that of the native enzymes $[8,9]$. The results obtained with glycollate and described earlier clearly show that $\mathrm{N}(5)$ covalent adducts are viable intermediates in the catalytic dehydrogenation of glycollate. that this deduction can be extrapolated to the mechanism of dehydrogenation of L-lactate is not certain in all its details, but we think it reasonable to assume, that the same basic mechanism will be operative within this family of enzymes and substrates. As discussed in detail elsewhere [4,12], the covalent N(5) adduct might be a true intermediate only in the case of glycollate, where the steric requirements are lower than for lactate. With the latter the adduct might be a transition state, i.e. formation of the N(5)$\mathrm{C} \alpha$ bond might be incomplete and be concerted with fragmentation. In fact, the crystal structures of $b_{2}$ and GO suggest the possibility of steric overcrowding in the substrate binding site. With both enzymes the peptide chain comes close to the flavin Re face, with Ala198 in b 2 and Ala 79 in GO being the nearest residue to the flavin N(5) position. In LO the homologous residue is Gly99. The distance between $\mathrm{N}(5)$ and the $\mathrm{CH}_{3}$ group of Ala79 in $\mathrm{GO}$ is $4.6 \AA$. If the arrangement of the protein around the flavin is similar in $\mathrm{LO}$ to that in $\mathrm{b}_{2}$ and $\mathrm{GO}$, there should be no overcrowding between Gly99 and the glycollyl $\alpha$ H, permitting the formation of the observed flavin N(5)-glycollyl adducts. However, with lactate as substrate steric crowding introduced by the methyl residue may prevent the formation of a stable lactoyl adduct. Also, in a putative lactoyl-N(5) adduct in the active center of either $\mathrm{GO}$ or $\mathrm{b}_{2}$, steric overcrowding seems probable, and would result in destabilization of the adduct. It is conceivable that evolution has created conditions in which the transition state/intermediate is not stabilized too much in order to promote catalysis. Furthermore, work with chemical models has shown that overcrowding plays a crucial role in the stability of $N(5)$ adducts. Thus, the adduct of formaldehyde to flavin $\mathrm{N}(5)$ is quite stable, while the corresponding one with acetaldehyde cannot be observed [15]. Similarly N(5) alkylated flavinium cations cannot be obtained when the substituent is 
isopropyl, while they are stable in the case of ethyl, and methyl [16].
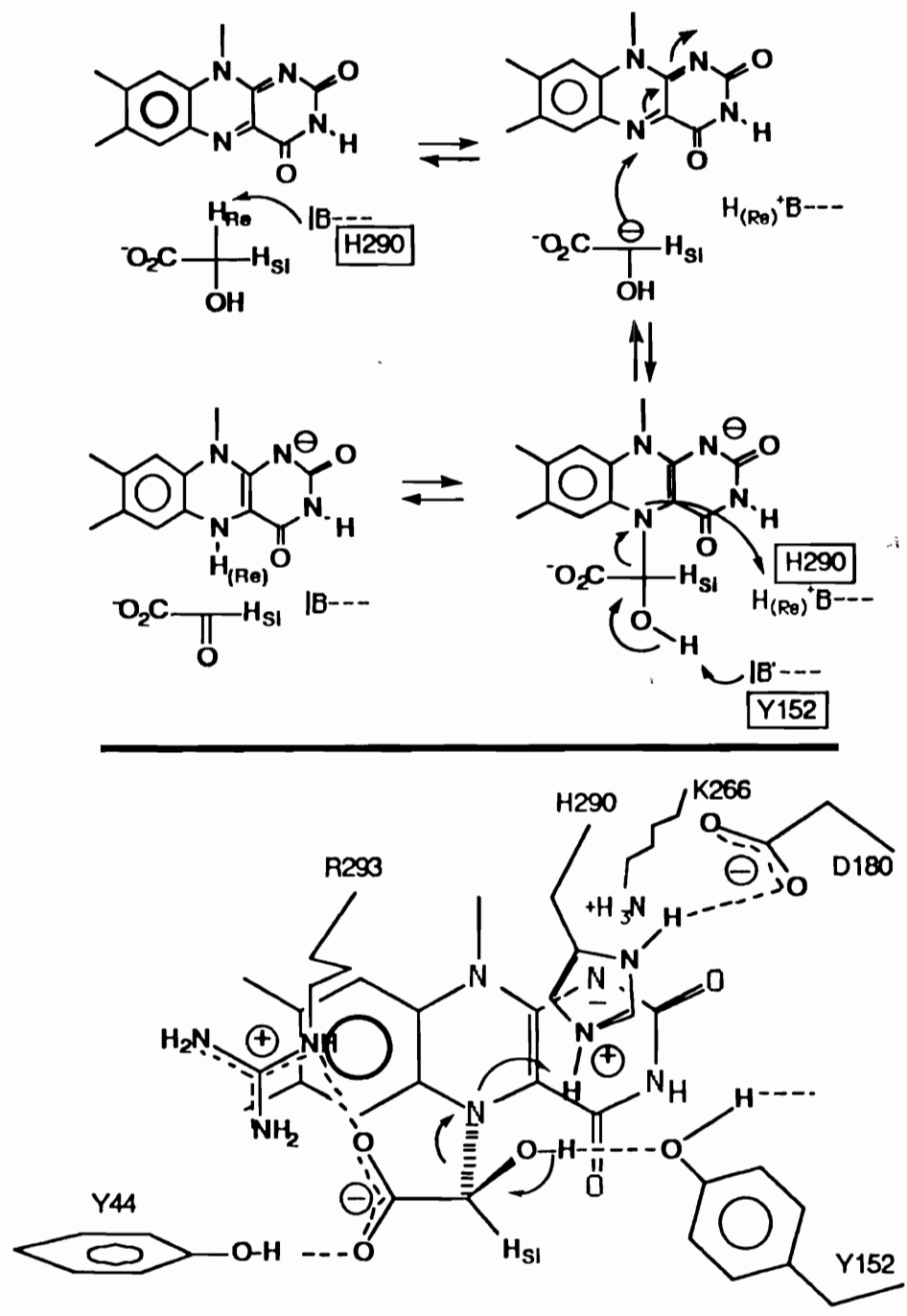

Figure 4 (A), top panel: Reaction of glycollate involving abstraction of $\mathrm{H}_{\mathrm{Re}}$ and formation of a labile covalent adduct which can decay to reduced enzyme and glyoxylate. Note that in this case the interaction of the glycollate $\alpha-\mathrm{OH}$ of the adduct with Tyr152. promotes the decay. 


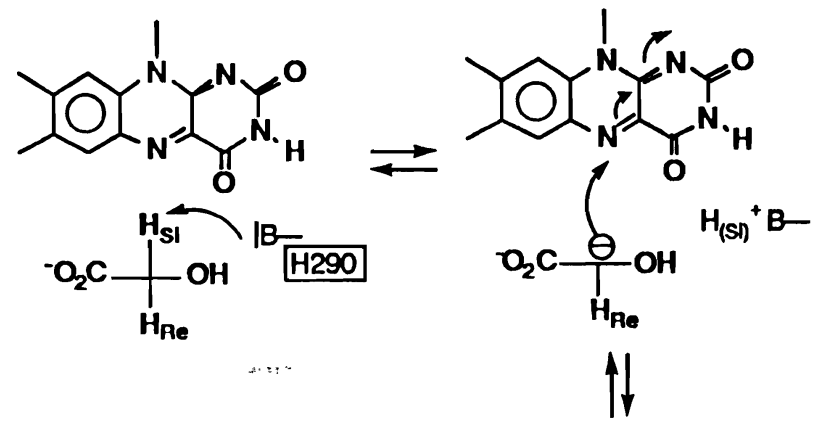<smiles></smiles>

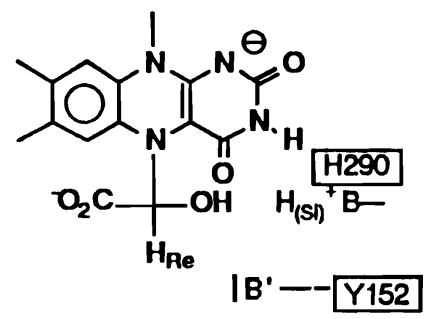

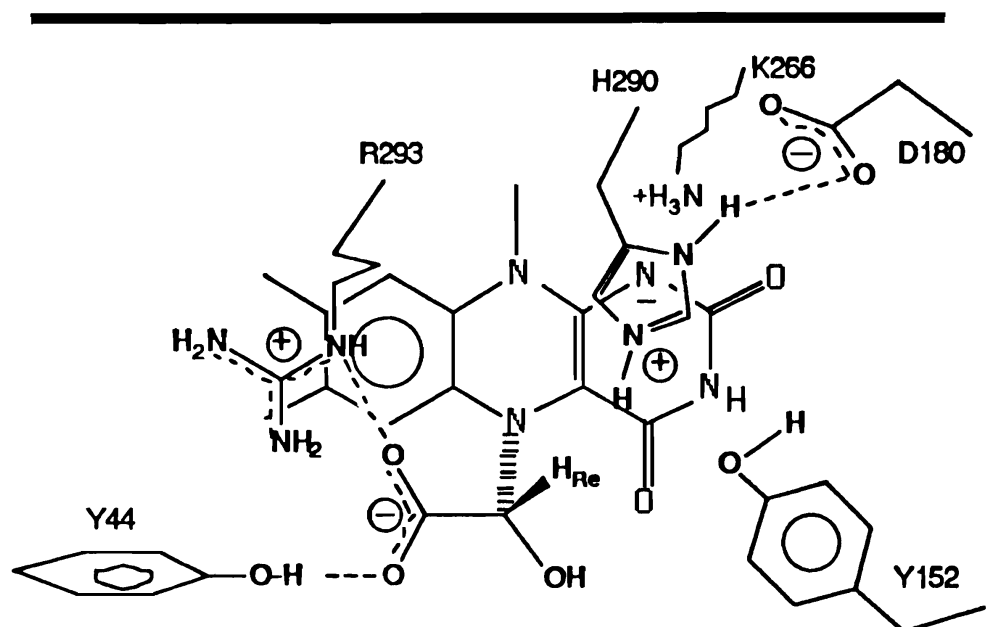

Figure 4: (B), top panel: mode of interaction of glycollate with the active center of LO. Note that the glycollate $\mathrm{H}_{\mathrm{Si}}$ reacts and leads to formation of a covalent adduct, which does not decay to reduced enzyme and glyoxylate. Lower panel: proposed structure of the stable covalent intermediate and interaction with active center functional groups. Note the absence of interaction of the glycollate $\alpha-\mathrm{OH}$ with Tyr 152 . 
Acknowledgements: We acknowledge many interesting discussions with Dr. F. Lederer on mechanistic topics, and financial support from the Deutsche Forschungsgemeinschaft to SG and from the U.S. Public Health Service (GM 11106:) to VM.

\section{References:}

1. Giegel, D.A., Williams, C.H.,Jr., and Massey, V. 1990. J.Biol.Chem. 2656626-6632

2. Lederer, F. and Mathews, F.S. 1987. in Flavins and Flavoproteins (Edmondson, D.E. and McCormick, D.B. eds) pp 133-142, de Gruyter, Berlin,

3. Lindqvist, Y., and Branden, C.I. 1989. J.Biol.Chem. 264, 3624-3628

4. Ghisla, S., and Massey, V., 1991. in "Chemistry and Biochemistry of Flavoproteins" (Müller, F., ed), CRC press, Inc, in press

5. Manstein, D.J., Massey, V., Ghisla, S. and Pai, E.F. 1988. Biochemistry 27, 2300-2305

6. Manstein, D.J., Pai, E.F., Schopfer, L.M. and Massey, V. 1986. Biochemistry 25, 6807-6816

7. Lederer, F., 1991. in "Chemistry and Biochemistry of Flavoproteins" (Müller, F., ed), CRC press, Inc, in press

8. Reid, G.A., White, S., Black, M.T., Lederer, F., Mathews, F.S. and Chapman, S.K. 1988. Eur. J. Biochem. 178, 329-333

9. Macheroux P., Massey, V., Thiele, D.J., Söderlind, E., and Lindqvist, Y., this volume

10. Ghisla, S., and Massey, V., 1975. J. Biol. Chem. 250, 577-584

11. Ghisla, S., and Massey, V. 1977. J. Biol. chem. 252, 6729-6735

12. Ghisla, S., and Massey, V.1989. Eur. J. Biochem. 181, 1-17

13. Massey, V., Ghisla, S., and Kieschke, K., 1980. J. Biol. Chem. 255, 2796-2806

14. Ghisla, S., and Massey, V. 1980. J. Biol. Chem. 255, 5688-5696

15. Blankenhom, G., Ghisla, S., and Hemmerich, P. 1972. Z. Naturforschung 27B, 1038-1040

16. Ghisla, S., Hartmann, U., Hemmerich, P., and Müller, F., 1973. Liebig's Ann. Chem. 13881415 